\title{
Anterior and middle superior alveolar block anesthesia: A new technique estimated
}

\author{
Kubais $\mathrm{H}$ AL-ASSAF* \\ Ahmed A ABDUL - MAWJOOD** \\ Faiz A AL-SULTAN***
}

\begin{abstract}
In this study, an estimation of new technique to achieve anesthesia at anterior and middle superior alveolar nerve through palatal injection was performed.

Total (25) patients who need conservative dental treatment were received this injection for treatment of (39) teeth. Success rate recorded in $(72 \%)$ of patient who satisfy this new injection technique completely and (20\%) failure rate reported ( $8 \%$ ) of patients reported pain during dental work but did not need supplementary anesthesia. The complication recorded as follows; (15) patients feel pain immediately during injection, (4) patients had postoperative pain and (4) patients had swelling. In conclusion it is recommended that this new technique to be assessed clinically for other dental work and comparison with other technique utilized.
\end{abstract}

Key Words: Nerve block, local anesthesia.

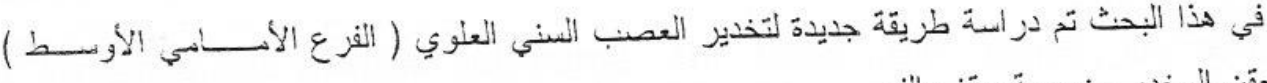

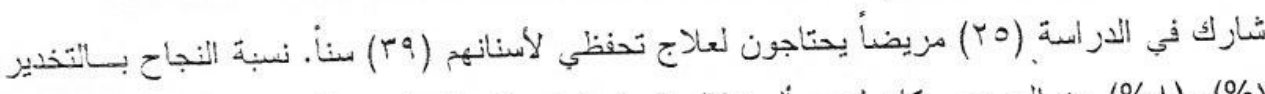

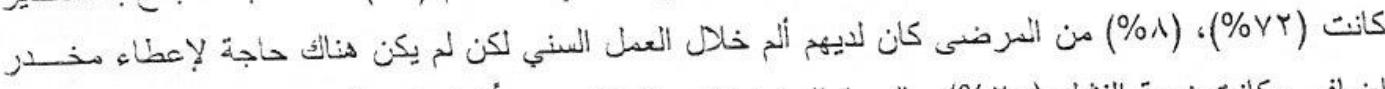

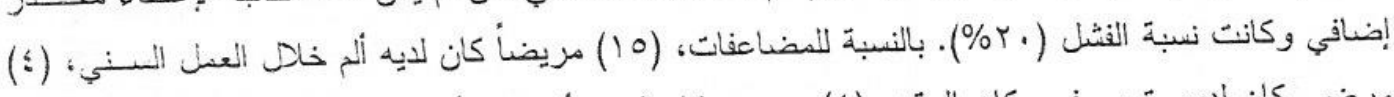

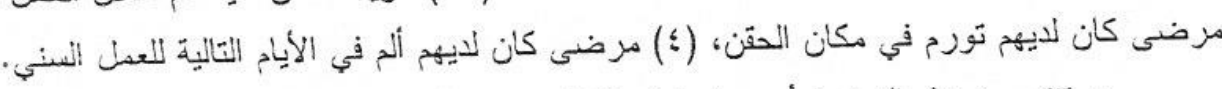

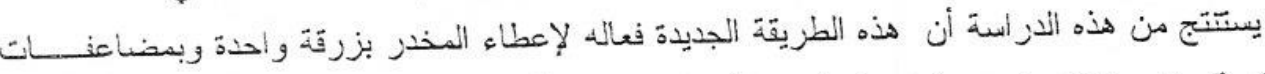

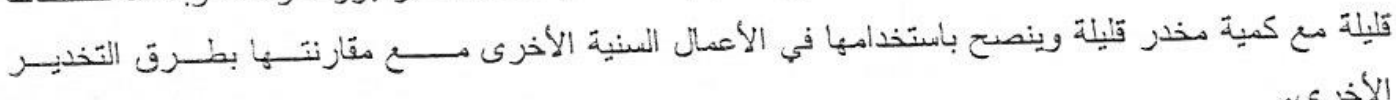

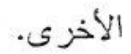

* Kubais Hajim AL - ASSAF; BDS, MSc: Assistant Lecturer. Department of Conservative Dentistry, College of Dentistry, University of Mosul, Mosul, IRAQ.

**Ahmed Abdul - Ilah A.3DUL - MAWJOOD; BDS, MSc: Assistant Lecturer. Department of Pedodontics. Orthodontics, \& Preventive Dentistry, College of Dentistry, University of Mosul. Mosul, IRAQ.

***Faiz Abdul - Razzak AL - SULTAN; BDS, MSc: Assistant Lecturer. Department of Oral \& Maxillofacial Surgery, College of Dentistry, University of Mosul, Mosul, IRAQ. 


\section{INTRODUCTION}

Supraperiosteal infiltration anesthesia was oldest technique to achieve anesthesia for maxillary teeth ${ }^{(1)}$. Although it is simple procedure to be accomplished successfully, there are several valid reason to search for other techniques such as
nerve block when ever its possible these includes: -

1. Infiltration anesthesia will produce anesthesia for adjacent soft tissue such as lip and check which make patient uncomfortable as well as dentist will loose esthetic smile
line consideration during dental treatment ${ }^{(2)}$. 2. It is contraindicated in case of presence of acute infection at site of injection to avoid
spread of infection.

3. In case of multiple teeth treatment to avoid numerous needle penetration that may produce pain to patient as well as to avoid administration of large volume of drug
with increase risk of systemic complications.

4. In case of failure to achieve anesthesia due to long root or due to presence of dense bone covering the root especially at the site of upper central incisor ${ }^{(3)}$.

The development of anterior and middle superior alveolar block through infra orbital parasthesia in the lip and check and it is contraindicated in bresent it still cause soft tissue at injection site (1). Recent study show that infra orbital presence of acute infection and only small percentage of dentist performed it because block not enjoys popularity as fear from eye injury ${ }^{(4)}$.

In the following study a new technique to achieve anesthesia for anterior and middle superior alveolar (AMSA) nerve through palatal approach were estimated. This technique overcome all the problem related to infiltration technique as it produce no anesthesia for infra orbital nerve (no soft tissue parasthesia), need single injection at labial or buccal site ${ }^{(2.5 .6)}$.

This new technique introduced at 1997 by the development of special electronic computerized system (Wand system) for administration of local anesthesia. Although this new technique performed using this special system at the begin but it is possible to be administered using conventionally used dental syringe providing that ultra short needle used to ensure proper orientation of the needle and to maintain steady control of pressure over syringe without bending of needle ${ }^{(7)}$.

This technique based on the fact that the bone of palate at premolar region represents nearest point to the anterior wall of maxillary sinus through which anterior and middle superior alveolar nerve pass. Injection of adequate amount of anaesthetic solution at that site sub periostealy will penetrate deeply to cause anesthesia for both nerves ${ }^{(2)}$. The landmark for the injection therefore is not an exact foramen, but rather between palatal suture and free gingival margin (Figure 1). 

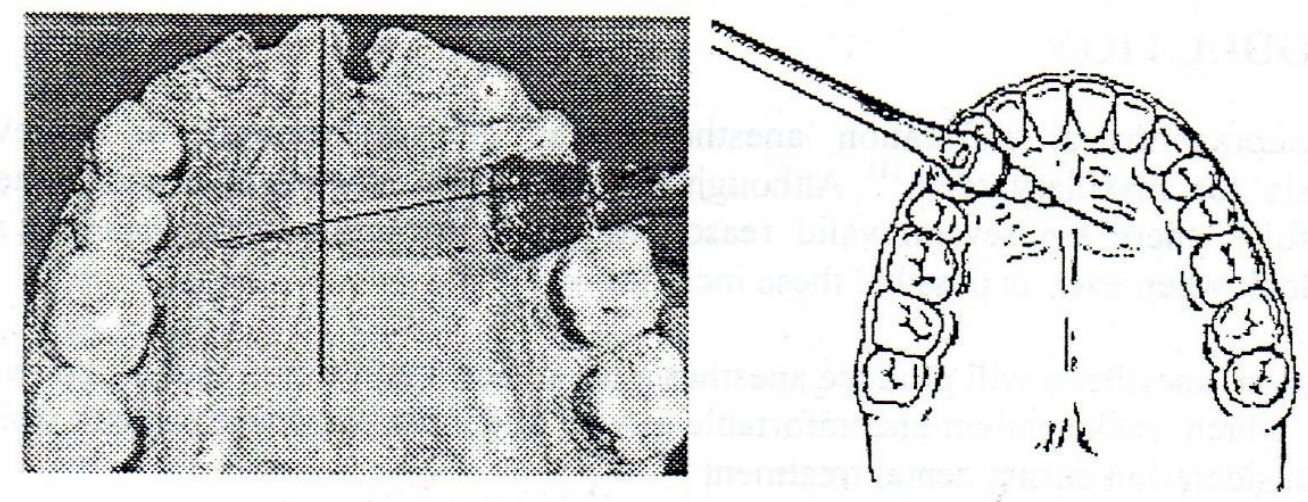

Figure (1): Anatomical landmarks for AMSA injection

The basic component is to make injection of anaesthetic solution maintained regularly with slow rate and (1.8) $\mathrm{ml}$ of solution used. Since palatal mucosa has adequate elasticity to maintain solution in contact with bone deeply. This ensure penetration of solution through palatal bone to reach neural tissue and blood vessel without being absorbed by soft tissue of the palate. So it is clinically as intra osseous injection $^{(2)}$.

In this study patient present for conservative treatment of their maxillary teeth (from central incisor to second premolar) receive this injection to be estimated by conventional syringe. Patients who need tooth extraction or surgery were excluded, as supplementary labial anesthesia needed which may produce misinterpretation with the result.

\section{MATERIALS AND METHODS}

The clinical study performed at university of Mosul/ College of Dentistry/ Department of conservative dentistry from (10) Oct. to (20) Nov. (2001).

All the patient presented to the clinic who need routine conservative treatment for their maxillary anterior and premolar teeth participated in the study after a verbal consent obtained for their approval. Patient who had systemic disease excluded from the study. Twenty-five patients participated in the study with age range between (1837) years.

Xylocaine (2\%) with $(1: 80000)$ concentration of adrenaline injected in this study. Low concentration of vasoconstrictor used to avoid necrosis and sloughing as that site had low vascularity [concentration of adrenaline should be $(1: 80000)$ or less and concentration of (1:50000) should be avoided ${ }^{(8)}$.

Each patient receives this injection technique at either left or right side as needed. Following protocol performed for each patient:

1 -Inform the patient for slow injection technique.

2-Place the chair at supine or semi-supine position.

3-Place topical anesthesia if desired.

4-27-gauge ultra short needle ( $8 \mathrm{~mm}$ length) with bend directed parallel to palatal tissue at bisecting line between a premolars and between mid palatine suture and free gingival margin. 
5-Orient the needle at $45^{\circ}$ angle to the tissue, advance the needle very slowly to contact the bone, initiate slow rate of diffusion immediately after injection to anaesthetize the injection site and reduce the pain.

6-Place side of mirror at injection site forcibly to maintain pressure over injection site. Continue the injection until $1.8 \mathrm{ml}$ of solution has been deposited over 1 minute.

7-Remove the needle slowly and try to avoid excess of anesthetic solution dripping to the back of the mouth (Figure 2).

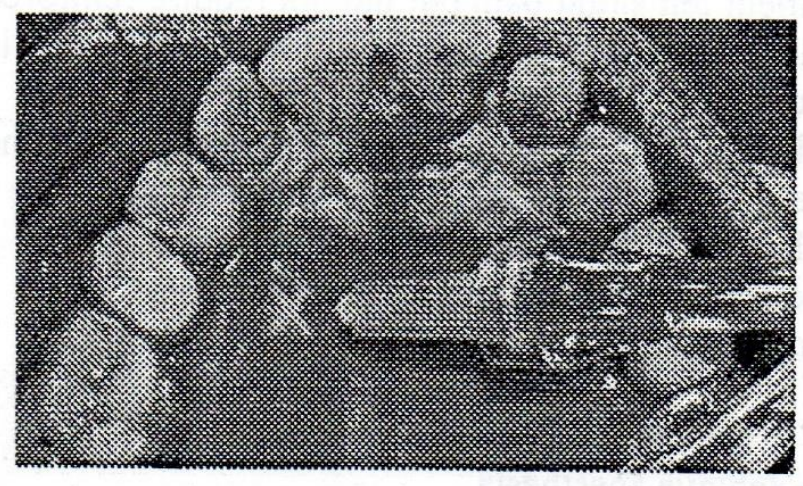

Figure (2): Injection of AMSA

For each patient the following data was collected (Figure 3):

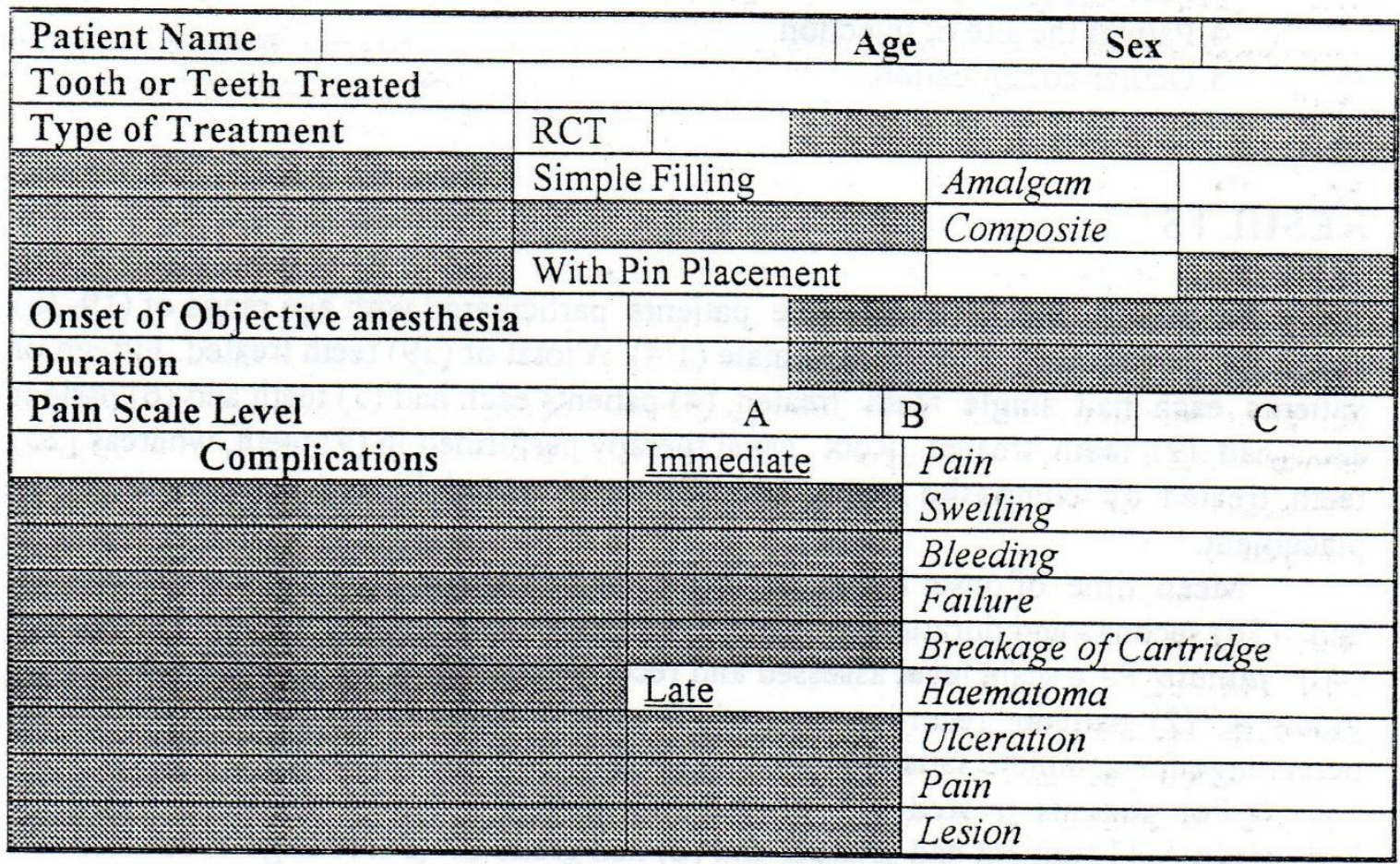

Figure (3): Patients records

The type of treatment performed either patient receives simple operative treatment (Amalgam or composite filling with or without pin placement) or root canal therapy was performed. 
Onset of objective anesthesia recorded by placing sharp end of probe in gingival sulcus palatally down to the bone surrounding both upper lateral incisor and first premolar at injection site and recording the time at which complete abolish of pain obtained ${ }^{(9)}$. Duration recorded by asking the patient about the feeling of palatal anesthesia and recorded at next visit.

Assessments of grade of pain performed after operative treatment according to Dobb and Devier system according to following scale:

Grade A: Completely satisfactory anesthesia.

Grade B: The patient felt slight pain but did not require reinforcement

Grade C: Feeble anesthesia, another injection needed and in such case local infiltration performed ${ }^{(10)}$

Regarding the complication the record performed either after operative treatment immediately or later on at the follow up visit after one week. patient who did not return for follow up visit excluded from the study.

Immediate expected complications include ${ }^{(1,2)}$ : -

1.Pain during injection.

2. Swelling.

3. Bleeding at the site of injection.

4. Failure to achieve anesthesia.

5. Breakage of cartridge.

Late expected complications include: -

1. Haematoma formation or ecchymosis.

2. Ulceration or sloughing.

3. Post injection lesion or infection.

4. Pain at the site of injection.

5. Ocular complication.

\section{RESULTS}

In present study, twenty-five patients participated with age range of (19-38) year and percentage of male to female (1:4). A total of (39) teeth treated. Fifteen of patients each had single teeth treated, (4) patients each had (3) teeth and (6) patient each had (2) teeth treated. Root canal therapy performed in (9) teeth, whereas (23) teeth treated by composite filling and (7) teeth treated by amalgam with (5) pin placement.

Mean time of onset for objective anesthesia assessed palataly was (3) minutes and (50) seconds and duration of palatal anesthesia maintained for about (1) hour and (40) minute. Pain scale level assessed and recorded as follow; 18 patients recorded as grade A, (2) patients recorded as grade B and (5) patients recorded as grade $C$ with percentage for complete satisfactory or successful anesthesia recorded as (72\%).

For patients treated by root canal therapy pain records as follow; (6) patient had grade A, (1) patient had grade B and (2) had grade C. (Percentage of success rate was $66.66 \%$ ).

For patients receive simple operative treatment pain scale recorded as follow; (12) patient had grade A, (1) patient had grade B and (3) patient had grade C (Percentage of success rate was 75\%). All (5) patient who had pin placement in vital teeth had grade A pain scale. (Table 1 and Figures 3-5). 
Table (1): Represent number and percentage of patients according to pain scale

\begin{tabular}{|c|c|c|c|}
\hline $1-1 / 2$ & 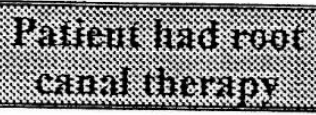 & Ifferfo & (1) \\
\hline 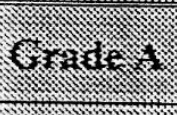 & $\begin{array}{c}6 \\
66.6 \% \\
\end{array}$ & $\begin{array}{c}12 \\
75 \% \\
\end{array}$ & $\begin{array}{c}18 \\
72 \% \\
\end{array}$ \\
\hline 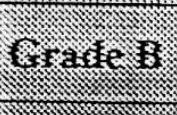 & $\begin{array}{c}1 \\
11 \% \\
\end{array}$ & $\begin{array}{c}1 \\
6 \% \\
\end{array}$ & $\begin{array}{c}2 \\
8 \% \\
\end{array}$ \\
\hline (x) & $\begin{array}{c}2 \\
22 \% \\
\end{array}$ & $\begin{array}{c}3 \\
19 \% \\
\end{array}$ & $\begin{array}{c}5 \\
20 \% \\
\end{array}$ \\
\hline
\end{tabular}

Grade A: Completely satisfactory anesthesia.

Grade B: The patient felt slight pain but did not require reinforcement

Grade C: Feeble anesthesia, another injection needed and in such case local infiltration performed

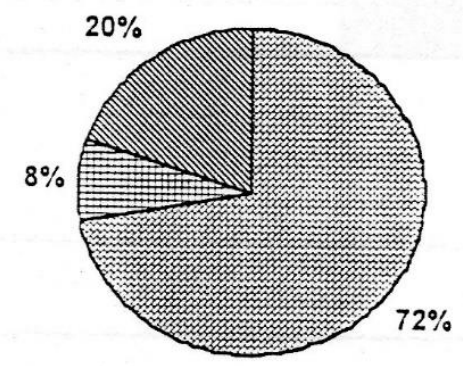

Figure (3): Percentge of different pain scale in both groups

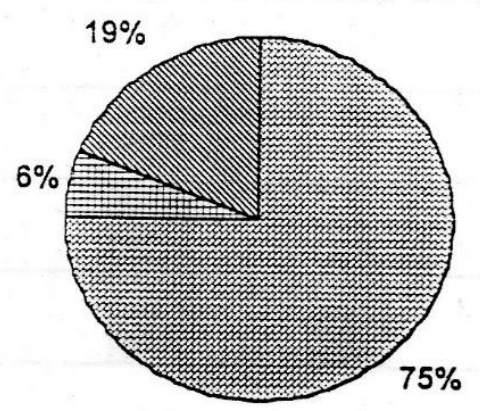

Figure (4): Percentge of different pain scale in group treated with simple operative traetment

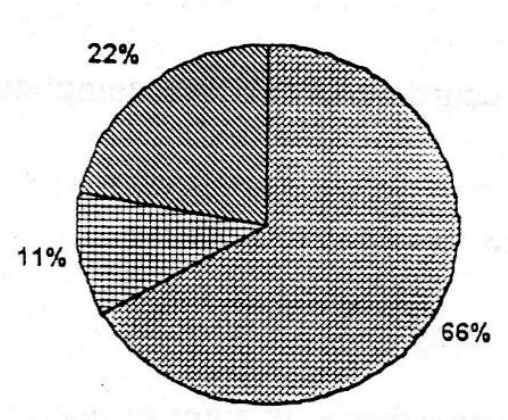

Figure (5): Percentge of different pain scale in group treated with root canal therapy 
Complications recorded as follow; for immediate complications, pain during injection in (15) patients, swelling in (4) patients, bleeding at injection site in (2) patients and failure to achieve anesthesia in (5) patients. For late complications, haematoma formation in (3) patients and pain at injection site in (4) patients (Table 2 and Figure 7)

Table (2): Complications after AMSA injection

\begin{tabular}{|c|c|c|}
\hline 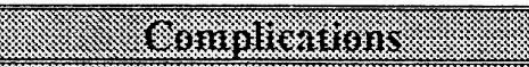 & 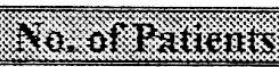 & 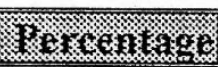 \\
\hline (1) & 15 & $60 \%$ \\
\hline 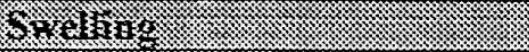 & 4 & $16 \%$ \\
\hline 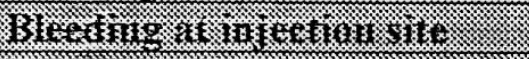 & 2 & $8 \%$ \\
\hline T & 5 & $20 \%$ \\
\hline 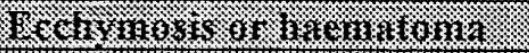 & 3 & $12 \%$ \\
\hline 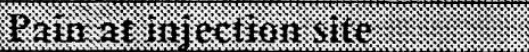 & 4 & $16 \%$ \\
\hline
\end{tabular}

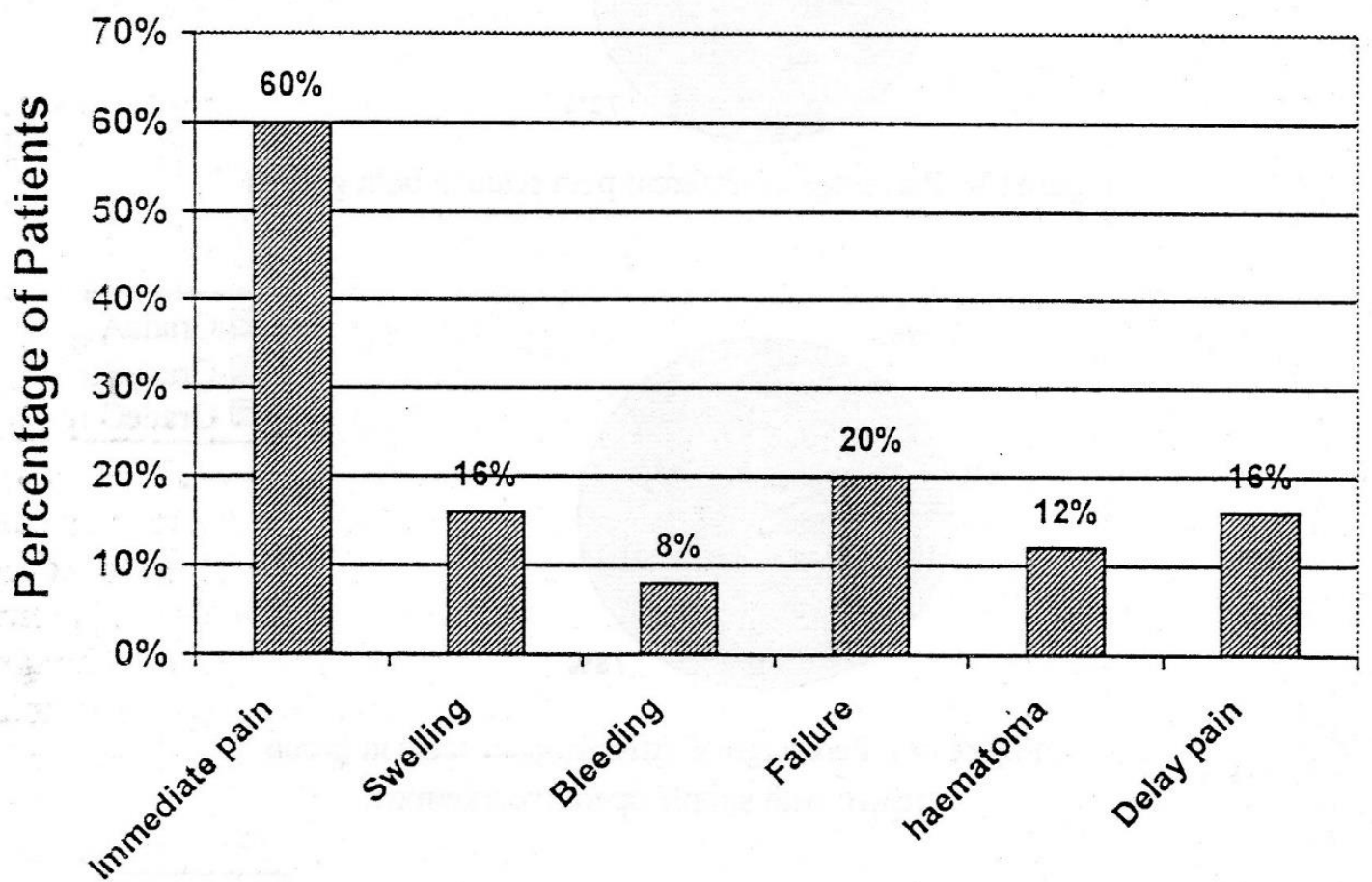

Figure (7): Percentage of patients according to different complication after AMSA injection

\section{DISCUSSION}

The achievement of pulp anesthesia in anterior maxillary region is of primary importance. This because local infiltration (sub periosteal) produce soft tissue parasthesia that is not desired by the patient as well as the dentist who may need to asses lip smile line for esthetics consideration which may lost by anesthesia to the 
muscle of facial expression. Other disadvantages includes needed of multiple injection for multiple teeth treatment, contraindication in case of infection at injection site as well as failure when dens overlaying bone existed ${ }^{(1-3)}$. This new technique used in present study overcomes these difficulties and satisfactory anesthesia achieved in
about $(72 \%)$ of cases.

Although the technique recommended to be used by the Wand electronic system but in our study it could be assumed to be used with ultra short needle on ordinary syringe and pressure on injection site by other metallic instrument to achieve slow rate
of diffusion through the bone.

Other advantage of the technique it's simplicity since landmark determination was simple and no true foramina existed ${ }^{(5)}$. It is in fact on intra osseous injection due to elastic palatal mucosa that maintains the solution deeply in contact with bone. So that injection of adequate amount of solution (at least $1.8 \mathrm{ml}$ ) is mandatory to maintain the pressure and ensure reaching the solution to somewhat a remote area for nerve block ${ }^{(2)}$. In this study higher success rate obtained in group treated by simple operative therapy compared to those receive root canal therapy (75\% \& $66 \%$ respectively). This result where expected since lowering pain threshold in patient who had tooth ache and need root canal therapy may elevate pain sensation ${ }^{(11)}$. Also in higher success patient who receive pin placement -which is such painful procedurehave previous toothache.

Delayed onset was expected since a remote block technique need an extra time for work ${ }^{(1)}$ In this study onset of objective anesthesia was about (3) minutes and (50)
second.

Regarding the complication, it was expected that the injection of such amount in such limited area produce pain in $(60 \%)$ of patient but all of whom are stated that it is mild tolerable pain due to slow injection procedure. Swelling that developed resolved in (3) cases without complication at next visit and only one patient had persistent ecchymosis at next visit after (1) week that resolved later on (after 2 week). Failure to achieve anesthesia may simply due to the fact that shallow palatal vault may play role and farther study for such anatomical variation suggested ${ }^{(12)}$. Also in one patient whom upper second premolar need treatment failure may attributed to the lack of middle superior alveolar nerve and in such case pulpal innervation of premolars shared by both anterior and posterior nerves. Other complications -although not recorded but its recommend to be avoided- which is highly expected including cartridge breakage by forceful injection, ulceration or sloughing post injection lesion
and ocular complications ${ }^{(1,2)}$.

Finally this technique recommended to be used routinely for multiple operative work as well as to be used as supplementary technique for patient undergoing apical surgery as prolong anesthesia may provoke recovery and further injection at that site may fail to produce adequate anesthesia. Therefore this remote block technique could be regarded as supplementary anesthetic technique during operation.

\section{REFERENCES}

1. Malamed SF. Handbook of Local Anesthesia. $2^{\text {nd }}$ Edn. Mosby Company. 1997.

2. Friedman MJ, Hochman MN. The AMSA injection: anew concept for local anesthesia of maxillary teeth using a computer - controlled injection system. Quint
Int. 1998; 29(5): 297-303. 
3. Kaufman E, Weinstein P, Milgrom P. Difficulties in achieving local anesthesia. $J$ Am Dent Assoc. 1984; 108: 205-208.

4. Hamid RS, AL-Sandook TA, Fathie WKh. The palatal injection: A painless approach. Al-Rafidain Dent J. 2001; 1(Sp Iss): 379-384.

5. Arlen DL. Advancement in the delivery of local anesthesia. Practical Periodontal and Esthetic Dentistry. 1998; 10(9): 1194 (Abstract).

6. Levato H. Giving the wand a shot. Dental Practice and Finance. 1998 (Abstract).

7. Wand User Communication. Reviewing the AMSA: Available injection for everyday practice. Wand User Communication. 1999; 13: 1-3 (Abstract).

8. The Wand Manual. AMSA technique. The Wand Manual. 2000: 1-2 (Abstract).

9. Agren $\mathrm{E}$ Danelson K. Conduction block analgesia in the mandible. A comparative investigation of the technique of Fischer and Gow-Gates. Swed Dent J. 1981; 5: 8184

10. Dobo EC, Devier CL. L-arterenol as vasoconstrictor in local anesthesia. J Am Dent Assoc. 1950; 40(4): $43-436$.

11. Jastak T, Yagiela JA, Donaldson D. Local Anesthesia of Oral Cavity. $1^{\text {st }}$ Edn. WB Saunders Co. 1995.

12. Steven P. Is the wand so wonderful? LVI Dental Vision. 1998; 7 (Abstract). 